QUARTERLY OF APPLIED MATHEMATICS

VOLUME LXVIII, NUMBER 4

DECEMBER 2010, PAGES 645-660

S 0033-569X(2010)01168-X

Article electronically published on September 21, 2010

\title{
LAPLACE'S EQUATION IN THE EXTERIOR OF A CONVEX POLYGON. THE EQUILATERAL TRIANGLE
}

\author{
BY \\ A. CHARALAMBOPOULOS (Department of Material Science and Engineering, University of \\ Ioannina, Greece), \\ G. DASSIOS (Department of Applied Mathematics and Theoretical Physics, University of Cambridge, \\ United Kingdom), \\ AND
}

\begin{abstract}
A. S. FOKAS (Department of Applied Mathematics and Theoretical Physics, University of Cambridge, United Kingdom)
\end{abstract}

Abstract. A general method for studying boundary value problems for linear and for integrable nonlinear partial differential equations in two dimensions was introduced in Fokas, 1997. For linear equations in a convex polygon (Fokas and Kapaev (2000) and (2003), and Fokas (2001)), this method: (a) expresses the solution $q(x, y)$ in the form of an integral (generalized inverse Fourier transform) in the complex $k$-plane involving a certain function $\hat{q}(k)$ (generalized direct Fourier transform) that is defined as an integral along the boundary of the polygon, and (b) characterizes a generalized Dirichlet-toNeumann map by analyzing the so-called global relation. For simple polygons and simple boundary conditions, this characterization is explicit. Here, we extend the above method to the case of elliptic partial differential equations in the exterior of a convex polygon and we illustrate the main ideas by studying the Laplace equation in the exterior of an equilateral triangle.

Regarding (a), we show that whereas $\hat{q}(k)$ is identical with that of the interior problem, the contour of integration in the complex $k$-plane appearing in the formula for $q(x, y)$ depends on $(x, y)$. Regarding (b), we show that the global relation is now replaced by a set of appropriate relations which, in addition to the boundary values, also involve certain additional unknown functions. In spite of this significant complication we show that, for

Received January 29, 2009 and, in revised form, February 18, 2009.

2000 Mathematics Subject Classification. Primary 35C15, 35J05, 35J25.

Key words and phrases. Laplace equation, equilateral triangle, exterior domain.

The second author is on leave from the University of Patras and ICE-HT/FORTH Greece. His current address is the Division of Applied Mathematics, Department of Chemical Engineering, University of Patras, GR 26504 Patras, Greece.

The present work was performed under the Marie Curie Chair of Excellence Project BRAIN, granted to the second and the third author by the European Commission under code number EXC 023928.

(C)2010 Brown University 
certain simple boundary conditions, the exterior problem for the Laplace equation can be mapped to the solution of a Dirichlet problem formulated in the interior of a convex polygon formed by three sides.

1. Introduction. Constructing analytic solutions for Laplace's equation in the exterior of a polygon is a long-standing open problem in the theory of partial differential equations. On the other hand, a method for solving Laplace's equation in the interior of a convex polygon was presented in [2]. This is a particular case of a general method for linear boundary value problems for open or closed convex polygons introduced by the third author in [3. The basic elliptic equations in an equilateral triangle for a large class of boundary conditions were studied in 1. In the above works, convexity played a crucial role, and this is the reason for analyzing interior problems. In the present work we investigate the solution of an exterior problem. In order to elucidate the effects of non-convexity we restrict our analysis to the simple case of Laplace's equation.

We first show that the integral representation for the exterior solution, in addition to a change of sign which reflects the change of direction of the normal to the boundary, differs from the analogous representation of the interior problem in the following important way: For the interior problem for a convex $n$-polygon, the integral representation involves the integrals of the $n$ functions $\left\{\hat{q}_{j}(k)\right\}_{j=1}^{n}$ along $n$ specific rays in the complex $k$-plane. For the exterior problem, the integral representation involves the same functions $\left\{\hat{q}_{j}(k)\right\}_{j=1}^{n}$, but the rays of integration depend on $(x, y)$. Actually, the extensions of the sides of the polygon divide the plane into certain domains, and one of the rays of integration rotates by $\pi$ as the point $(x, y)$ moves into a neighboring domain. In other words, the exterior problem has a constant spectral form but a varying spectral representation.

For example, in the integral representation of the exterior solution for an equilateral triangle, the three rays of integration rotate by $\pi / 3$ as $(x, y)$ moves successively in the six convex domains generated by the three sides of the triangle. Thus, the rays return to their original positions after $(x, y)$ travels around the six domains.

For a convex polygon, the so-called Dirichlet-to-Neumann map is characterized by the global relation which is an equation coupling the Dirichlet and Neumann boundary values [5]. We show that, for problems formulated in the exterior of a convex polygon, the fundamental domain must be subdivided to an appropriate set of convex subdomains, and in each of these subdomains there exists an appropriate global relation. This construction introduces additional unknown functions which makes the characterization of the Dirichlet-to-Neumann map more complicated. In spite of this significant complication, we will show that if one prescribes the same function as a Dirichlet boundary condition on each side of an equilateral triangle and if this function is symmetric with respect to the midpoint of the side, then the exterior problem can be mapped to the solution of a Dirichlet problem formulated in the interior of a convex polygon formed by three sides. On the other hand, the general Dirichlet problem gives rise to a matrix Riemann-Hilbert problem.

In what follows, we will assume existence; however, it is possible to eliminate this assumption. In fact, it turns out that the global relation is not only a necessary but also a sufficient condition for existence. Indeed, combining the global relation with the 
integral representation mentioned earlier, one can prove that the question of existence of solution of a given boundary value problem can be reduced to the question of analyzing the global relation. This proof is not presented here, but it is similar with the proof of the analogous result for the interior problem given in 7 .

The paper is organized as follows. In Section 2 the integral representation for the exterior of an $n$-polygon is derived. In Section 3 this general result is illustrated for the particular case of the exterior of an equilateral triangle. Section 4 constructs the global relation in the case that the same function is prescribed as a Dirichlet boundary condition on each side of the triangle. Furthermore, it is shown that if this function is anti-symmetric with respect to the midpoint of the side, then the Dirichlet problem can be mapped to the solution of a Dirichlet problem formulated in the interior of a convex polygon formed by three sides.

2. The integral representation. Let $z_{1}, z_{2}, \ldots, z_{n}, z_{n+1}=z_{1}$ be the $n$ vertices of a bounded convex polygon $\Omega^{(i)}$ in the complex plane. Denote by $\Omega^{(e)}$ the domain exterior to $\Omega^{(i)}$ and by $\partial \Omega^{(i)}$ the boundary of $\Omega^{(i)}$.

Proposition 2.1. Suppose that the real-valued function $q(x, y)$ is a harmonic function in $\Omega^{(e)}$, which decays as $|x|+|y| \rightarrow \infty$ and which is smooth on the boundary $\partial \Omega^{(i)}$. Then

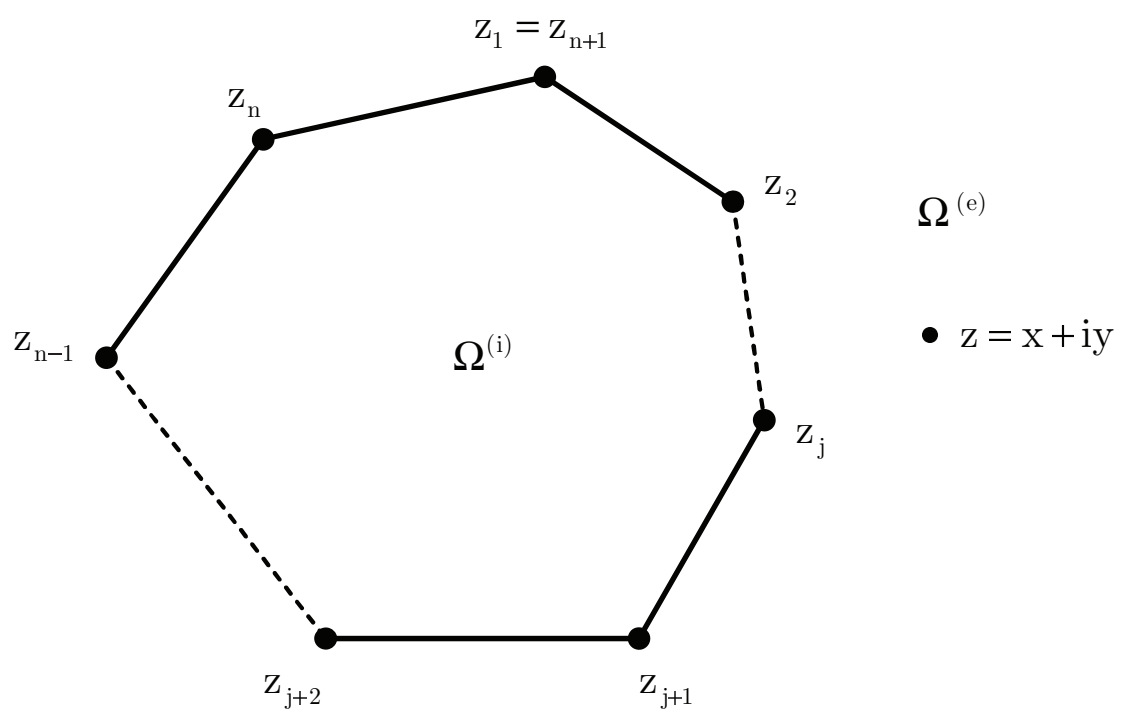

FIG. 2.1 
the following relations are valid:

$$
\begin{gathered}
\frac{1}{2 \pi i} \int_{\partial \Omega^{(i)}} \frac{\partial q\left(z^{\prime}\right)}{\partial z^{\prime}} \frac{d z^{\prime}}{z^{\prime}-z}= \begin{cases}0, & z \in \Omega^{(i)} \\
-\frac{\partial q(z)}{\partial z}, & z \in \Omega^{(e)}\end{cases} \\
\frac{\partial q(z)}{\partial z}=\left(\frac{1}{2 \pi i}\right)^{2} \int_{\partial \Omega^{(i)}}\left[\iint_{\mathbb{R}^{2}} \frac{e^{i k_{1}\left(x-x^{\prime}\right)+i k_{2}\left(y-y^{\prime}\right)}}{k_{1}+i k_{2}} d k_{1} d k_{2}\right] \frac{\partial q\left(z^{\prime}\right)}{\partial z^{\prime}} d z^{\prime}, \quad z \in \Omega^{(e)},
\end{gathered}
$$

where $z$ denotes the usual complex variable $z=x+i y$, and the integration is taken in the positive direction.

Proof. The equations

$$
z=x+i y, \quad \bar{z}=x-i y
$$

imply

$$
\partial_{z}=\frac{1}{2}\left(\partial_{x}-i \partial_{y}\right), \partial_{\bar{z}}=\frac{1}{2}\left(\partial_{x}+i \partial_{y}\right) .
$$

Hence, Laplace's equation becomes

$$
\frac{\partial^{2} q(z, \bar{z})}{\partial \bar{z} \partial z}=0
$$

which shows that the function $\partial_{z} q$ is analytic. Hence, using the fact that $q$ decays at infinity, Cauchy's theorem in the domain $\Omega^{(e)}$ yields equation (2.1). In the Appendix, we prove the following identity:

$$
\frac{1}{z-z^{\prime}}=\frac{1}{2 \pi} \iint_{\mathbb{R}^{2}} \frac{e^{i k_{1}\left(x-x^{\prime}\right)+i k_{2}\left(y-y^{\prime}\right)}}{i k_{1}-k_{2}} d k_{1} d k_{2} .
$$

Replacing in equation (2.1) $\frac{1}{z-z^{\prime}}$ by the RHS of equation (2.3) we find equation (2.2).

It was shown in [6] that, starting with representations of the type (2.2), it is possible to obtain Ehrenpreis-Palamodov-type representations by integrating with respect to $k_{N}$, where $k_{N}$ is the component of the vector $\mathbf{k}=\left(k_{1}, k_{2}\right)$, normal to the boundary. Using this approach, we will prove the following result.

Theorem 2.1. Under the assumptions of Proposition 2.1, the real-valued harmonic function $q$ admits the following integral representation:

$$
\frac{\partial q(z)}{\partial z}=-\frac{1}{2 \pi} \sum_{j=1}^{n} \int_{\hat{\ell}_{j}} e^{i k z} \widehat{q}_{j}(k) d k, \quad z \in \Omega^{(e)},
$$

where the spectral functions $\left\{\widehat{q}_{j}(k)\right\}_{j=1}^{n}$ are defined by

$$
\widehat{q_{j}}(k)=\int_{z_{j+1}}^{z_{j}} e^{-i k z} q_{z}(z) d z, \quad j=1,2, \ldots, n,
$$

and the rays $\hat{\ell}_{j}, j=1,2, \ldots, n$ emanating from the origin are defined as follows:

$$
\hat{\ell}_{j}=\left\{\begin{array}{ll}
\ell_{j}^{-}=\left\{k \in \mathbb{C}: \arg k=\pi-\arg \left(z_{j}-z_{j+1}\right)\right\}, & x_{N}^{j}<0 \\
\ell_{j}^{+}=\left\{k \in \mathbb{C}: \arg k=-\arg \left(z_{j}-z_{j+1}\right)\right\}, & x_{N}^{j}>0
\end{array},\right.
$$




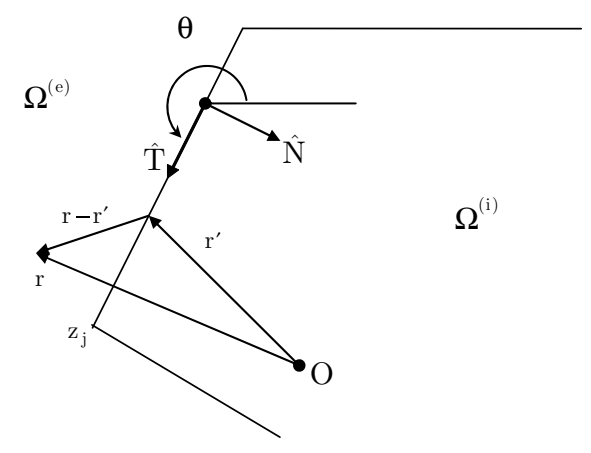

FIG. 2.2

where $x_{N}^{j}$ is the outward to $\Omega^{(e)}$ normal component of the vector $\mathbf{r}-\mathbf{r}^{\prime}$, with $\mathbf{r} \in \Omega^{(e)}$ and $\mathbf{r}^{\prime}$ on the $j$-th side. Finally,

$$
q(x, y)=2 \operatorname{Re} \int_{z_{0}}^{z=x+i y} \frac{\partial q\left(z^{\prime}\right)}{\partial z^{\prime}} d z^{\prime} .
$$

Proof. We introduce local coordinates on each side of the polygon in such a way that the normal $\widehat{\mathbf{N}}$ is exterior to $\Omega^{(e)}$, i.e. it points toward $\Omega^{(i)}$; see Figure 2.2. The Frenet system on the $j$-th side is

$$
\widehat{\mathbf{T}}=\frac{z_{j}-z_{j+1}}{\left|z_{j}-z_{j+1}\right|}, \quad \widehat{\mathbf{N}}=i \widehat{\mathbf{T}}
$$

We introduce the notations

$$
\mathbf{k}=\left(k_{1}, k_{2}\right), \quad \mathbf{r}=(x, y), \quad \mathbf{r}^{\prime}=\left(x^{\prime}, y^{\prime}\right)
$$

and we denote by $\left(k_{T}, k_{N}\right)$ and $\left(x_{T}, x_{N}\right)$ the Frenet components of $\mathbf{k}$ and of $\mathbf{r}-\mathbf{r}^{\prime}$, respectively, i.e.

$$
\mathbf{k}=k_{T} \widehat{\mathbf{T}}+k_{N} \widehat{\mathbf{N}}, \quad \mathbf{r}-\mathbf{r}^{\prime}=x_{T} \widehat{\mathbf{T}}+x_{N} \widehat{\mathbf{N}}
$$

Hence,

$$
k_{1}\left(x-x^{\prime}\right)+k_{2}\left(y-y^{\prime}\right)=k_{T} x_{T}+k_{N} x_{N}, \quad d k_{1} d k_{2}=d k_{T} d k_{N} .
$$

Let $\theta$ denote the argument of $z_{j}-z_{j+1}$; then

$$
k_{1}+i k_{2}=e^{i \theta}\left(k_{T}+i k_{N}\right) .
$$

Hence, in the local Frenet system, equation (2.2) becomes

$$
\frac{\partial q(z)}{\partial z}=-\frac{1}{4 \pi^{2}} \sum_{j=1}^{n} \iint_{\mathbb{R}^{2}}\left[\int_{z_{j+1}}^{z_{j}} \frac{e^{i\left(k_{T} x_{T}+k_{N} x_{N}\right)}}{k_{T}+i k_{N}} e^{-i \theta} \frac{\partial q\left(z^{\prime}\right)}{\partial z^{\prime}} d z^{\prime}\right] d k_{T} d k_{N} .
$$

Equation (2.8) implies

$$
x_{N}=\left(\mathbf{r}-\mathbf{r}^{\prime}\right) \cdot \widehat{\mathbf{N}} .
$$




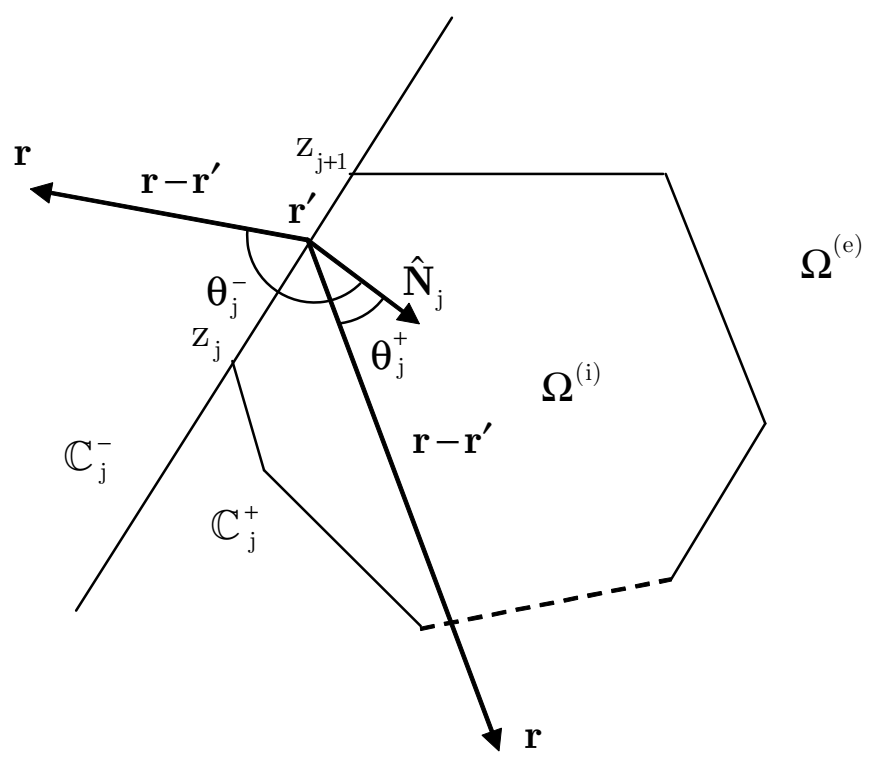

FIG. 2.3

In order to evaluate the integrals in the expression (2.9) we need to determine the sign of $x_{N}$. Hence, for each side of the polygon we define the splitting

$$
\begin{aligned}
& \mathbb{C}_{j}^{+}=\left\{\mathbf{r} \in \Omega^{(e)} \mid\left(\mathbf{r}-\mathbf{r}^{\prime}\right) \cdot \widehat{\mathbf{N}}_{j}>0\right\} \\
& \mathbb{C}_{j}^{-}=\left\{\mathbf{r} \in \Omega^{(e)} \mid\left(\mathbf{r}-\mathbf{r}^{\prime}\right) \cdot \widehat{\mathbf{N}}_{j}<0\right\}
\end{aligned}
$$

where $\mathbf{r}^{\prime}$ belongs to the $j$-th side of the polygon. Note that since the polygon is convex, $\mathbb{C}_{j}^{+}$is always a half-plane minus the polygon, while $\mathbb{C}_{j}^{-}$is a full half-plane.

Furthermore,

$$
\begin{aligned}
& \mathbf{r} \in \mathbb{C}_{j}^{+} \Rightarrow \theta_{j}^{+} \in\left(-\frac{\pi}{2}, \frac{\pi}{2}\right), \\
& \mathbf{r} \in \mathbb{C}_{j}^{-} \Rightarrow \theta_{j}^{-} \in\left(\frac{\pi}{2}, \frac{3 \pi}{2}\right) .
\end{aligned}
$$

a) The case where $\mathbf{r} \in \mathbb{C}_{j}^{-}$.

In this case $x_{N}<0$, and integrating in the lower $k_{N}$-half plane yields

$$
\int_{-\infty}^{\infty} \frac{e^{i k_{N} x_{N}}}{k_{T}+i k_{N}} d k_{N}= \begin{cases}-2 \pi e^{-k_{T} x_{N}}, & k_{T}<0 \\ 0, & k_{T}>0\end{cases}
$$

Therefore,

$$
-\frac{1}{4 \pi^{2}} \iint_{\mathbb{R}^{2}} \frac{e^{i\left(k_{T} x_{T}+k_{N} x_{N}\right)}}{k_{T}+i k_{N}} e^{-i \theta} d k_{T} d k_{N}=\frac{1}{2 \pi} \int_{0}^{+\infty} e^{-i k_{T}\left(x_{T}+i x_{N}\right)} e^{-i \theta} d k_{T} .
$$

Setting $k_{T}=k e^{i \theta}$, and using

$$
x_{T}+i x_{N}=\left(z-z^{\prime}\right) e^{-i \theta}
$$


we rewrite $(2.11)$ as

$$
\frac{1}{2 \pi} \int_{0 e^{-i \theta}}^{\infty e^{-i \theta}} e^{-i k\left(z-z^{\prime}\right)} d k=-\frac{1}{2 \pi} \int_{0 e^{i(\pi-\theta)}}^{\infty e^{i(\pi-\theta)}} e^{i k\left(z-z^{\prime}\right)} d k,
$$

where the last equality is obtained by replacing $k$ with $k e^{i \pi}$. Therefore,

$$
-\frac{1}{4 \pi^{2}} \iint_{\mathbb{R}^{2}} \frac{e^{i\left(k_{T} x_{T}+k_{N} x_{N}\right)}}{k_{T}+i k_{N}} e^{-i \theta} d k_{T} d k_{N}=-\frac{1}{2 \pi} \int_{\ell_{j}^{-}} e^{i k\left(z-z^{\prime}\right)} d k
$$

with

$$
\ell_{j}^{-}=\left\{k \in \mathbb{C} \mid \arg k=\pi-\arg \left(z_{j}-z_{j+1}\right)\right\} .
$$

b) The case where $\mathbf{r} \in \mathbb{C}_{j}^{+} \cap \Omega^{(e)}$.

In this case $x_{N}>0$, and integrating in the upper $k_{N}$-half plane yields

$$
\int_{-\infty}^{+\infty} \frac{e^{i k_{N} x_{N}}}{k_{T}+i k_{N}} d k_{N}= \begin{cases}0, & k_{T}<0 \\ 2 \pi e^{-k_{T} x_{N}}, & k_{T}>0\end{cases}
$$

Therefore,

$$
-\frac{1}{4 \pi^{2}} \iint_{\mathbb{R}^{2}} \frac{e^{i\left(k_{T} x_{T}+k_{N} x_{N}\right)}}{k_{T}+i k_{N}} e^{-i \theta} d k_{T} d k_{N}=-\frac{1}{2 \pi} \int_{0}^{\infty} e^{i k_{T}\left(x_{T}+i x_{N}\right)} e^{-i \theta} d k_{T} .
$$

Setting $k_{T}=k e^{i \theta}$, and using

$$
x_{T}+i x_{N}=\left(z-z^{\prime}\right) e^{-i \theta},
$$

we arrive at

$$
-\frac{1}{2 \pi} \int_{0}^{+\infty} e^{i k_{T}\left(x_{T}+i x_{N}\right)} e^{-i \theta} d k_{T}=-\frac{1}{2 \pi} \int_{0 e^{-i \theta}}^{\infty e^{-i \theta}} e^{i k\left(z-z^{\prime}\right)} d k
$$

Therefore,

$$
-\frac{1}{4 \pi^{2}} \iint_{\mathbb{R}^{2}} \frac{e^{i\left(k_{T} x_{T}+k_{N} x_{N}\right)}}{k_{T}+i k_{N}} e^{-i \theta} d k_{T} d k_{N}=-\frac{1}{2 \pi} \int_{\ell_{j}^{+}} e^{i k\left(z-z^{\prime}\right)} d k
$$

with

$$
\ell_{j}^{+}=\left\{k \in \mathbb{C} \mid \arg k=-\arg \left(z_{j}-z_{j+1}\right)\right\} .
$$

Hence, the proof of the Theorem is completed.

REMARK 2.2. Expressions (2.4) and (2.5) provide the generalized Fourier transform pair associated with Laplace's equation in the exterior domain $\Omega^{(e)}$. For a given point $z \in \Omega^{(e)}, \hat{\ell}_{j}$ can be fixed by studying the relative position of $z$ with respect to the side. The contours $\ell_{j}^{-}$(associated with the whole half-spaces) are exactly the same as the corresponding contours for the interior problem [1, whereas the contours $\ell_{j}^{+}$(associated with the half-spaces that contain the polygon) are the opposite of $\ell_{j}^{-}$. 


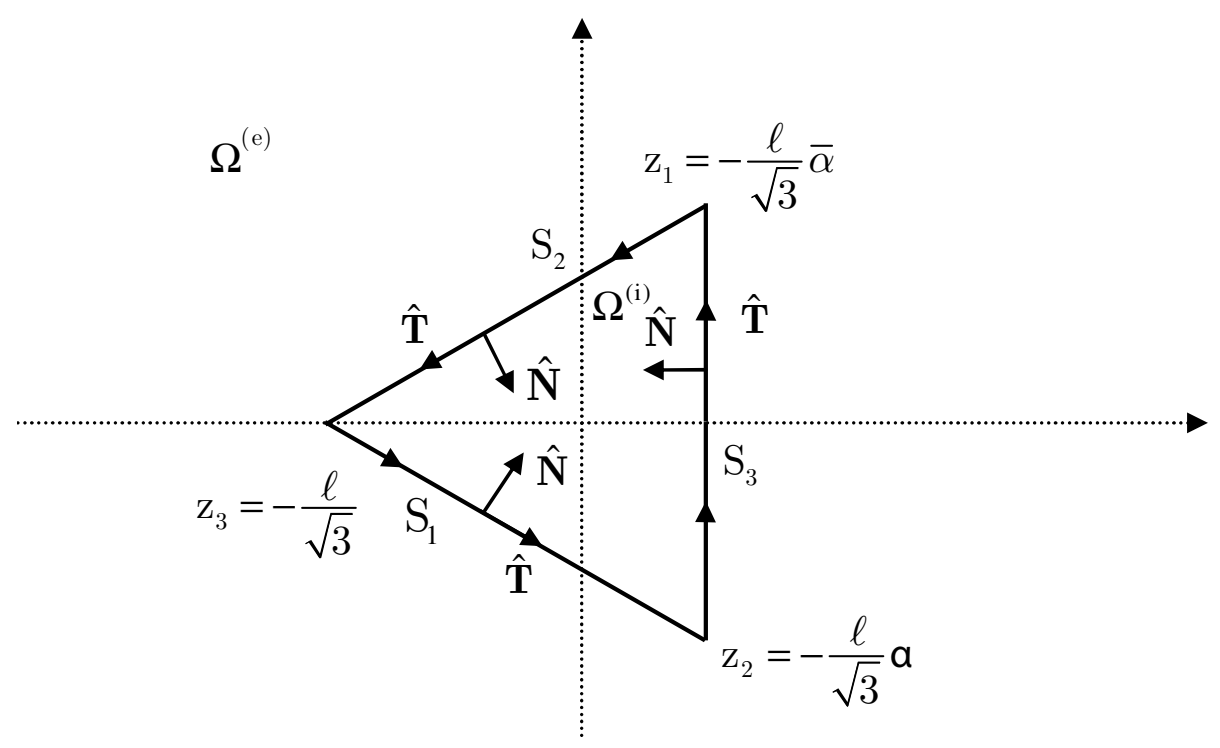

FIG. 3.1

3. The equilateral triangle. Set

$$
\alpha=e^{i \frac{2 \pi}{3}}=-\frac{1}{2}+i \frac{\sqrt{3}}{2}
$$

and let

$$
z_{1}=-\bar{\alpha} \frac{\ell}{\sqrt{3}}, \quad z_{2}=-\alpha \frac{\ell}{\sqrt{3}}, \quad z_{3}=-\frac{\ell}{\sqrt{3}}
$$

be the vertices of the equilateral triangle of side $\ell$, shown in Figure 3.1. The side $S_{j}$ is located opposite to the vertex $z_{j}, j=1,2,3$.

On the sides $S_{3}, S_{2}, S_{1}$, the parameterizations

$$
\begin{aligned}
& S_{3}: z(s)=\frac{\ell}{2 \sqrt{3}}+i s \\
& S_{2}: z(s)=\alpha\left(\frac{\ell}{2 \sqrt{3}}+i s\right) \\
& S_{1}: z(s)=\bar{\alpha}\left(\frac{\ell}{2 \sqrt{3}}+i s\right)
\end{aligned}
$$

where $-\frac{\ell}{2} \leq s \leq \frac{\ell}{2}$, imply

$$
\begin{aligned}
S_{3}: \partial_{z} & =-\frac{1}{2}\left(\partial_{N}+i \partial_{T}\right) \\
S_{2}: \partial_{z} & =-\frac{\bar{\alpha}}{2}\left(\partial_{N}+i \partial_{T}\right) \\
S_{1}: \partial_{z} & =-\frac{\alpha}{2}\left(\partial_{N}+i \partial_{T}\right)
\end{aligned}
$$


where $\partial_{N}$ and $\partial_{T}$ denote normal and tangential differentiation along $\widehat{\mathbf{N}}$ and $\widehat{\mathbf{T}}$. Let $q_{j}(s)$ and $\partial_{N} q_{j}(s)$ denote the Dirichlet and Neumann values of the side $S_{j}, j=1,2,3$, i.e.

$$
\left.q(x, y)\right|_{(x, y) \in S_{j}}=q_{j}(s),\left.\quad q_{N}(x, y)\right|_{(x, y) \in S_{j}}=q_{N_{j}}(s),-\frac{\ell}{2} \leq s \leq \frac{\ell}{2}, \quad j=1,2,3 .
$$

Then, the spectral functions $\widehat{q_{j}}(k)$ are defined by the following:

$$
\begin{aligned}
& \widehat{q_{3}}(k)=\int_{z_{2}}^{z_{1}} e^{-i k z} q_{z}(z) d z=\frac{1}{2}\left[-i E(-i k) \Psi_{3}(k)+E(-i k) \Phi_{3}(k)\right], \\
& \widehat{q_{2}}(k)=\int_{z_{1}}^{z_{3}} e^{-i k z} q_{z}(z) d z=\frac{1}{2}\left[-i E(-i \alpha k) \Psi_{2}(\alpha k)+E(-i \alpha k) \Phi_{2}(\alpha k)\right], \\
& \widehat{q_{1}}(k)=\int_{z_{3}}^{z_{2}} e^{-i k z} q_{z}(z) d z=\frac{1}{2}\left[-i E(-i \bar{\alpha} k) \Psi_{1}(\bar{\alpha} k)+E(-i \bar{\alpha} k) \Phi_{1}(\bar{\alpha} k)\right],
\end{aligned}
$$

where

$$
E(k)=e^{k \frac{\ell}{2 \sqrt{3}}}, \quad k \in \mathbb{C},
$$

$\left\{\Phi_{j}\right\}_{1}^{3},\left\{\Psi_{j}\right\}_{1}^{3}$ denote the Dirichlet and Neumann integrals,

$$
\begin{aligned}
& \Phi_{j}(k)=\int_{-\ell / 2}^{\ell / 2} e^{k s} \dot{q}_{j}(s) d s, \quad k \in \mathbb{C}, j=1,2,3, \\
& \Psi_{j}(k)=\int_{-\ell / 2}^{\ell / 2} e^{k s} q_{N_{j}}(s) d s, \quad k \in \mathbb{C}, j=1,2,3,
\end{aligned}
$$

and dot denotes differentiation. Indeed, introducing local coordinates, it follows that

$$
2 \widehat{q_{3}}(k)=\int_{z_{2}}^{z_{1}} e^{-i k z}\left(\partial_{x}-i \partial_{y}\right) q(x, y) d z=-i E(-i k) \Psi_{3}(k)+E(-i k) \Phi_{3}(k) .
$$

Similarly, the functions $\widehat{q_{2}}(k)$ and $\widehat{q_{1}}(k)$ can be obtained through rotations by $\frac{2 \pi}{3}$ and $\frac{4 \pi}{3}$ respectively. The extensions of the three sides of the triangle, partition the exterior of the triangle in the six regions denoted by $R_{11}, R_{22}, R_{33}, R_{21}, R_{32}, R_{13}$, as in Figure 3.2.

In summary, the integral representations for the exterior of the equilateral triangle depicted in Figure 3.1 are given by equation (2.4), where the spectral functions are defined by equations (3.4)-(3.9) and the appropriate rays (defined in (2.6)) in the particular domains are chosen as follows:

$$
\left(\widehat{\ell_{1}}, \widehat{\ell_{2}}, \widehat{\ell_{3}}\right)= \begin{cases}\left(\ell_{1}^{-}, \ell_{2}^{+}, \ell_{3}^{-}\right), & z \in R_{11} \\ \left(\ell_{1}^{+}, \ell_{2}^{+}, \ell_{3}^{-}\right), & z \in R_{13} \\ \left(\ell_{1}^{+}, \ell_{2}^{-}, \ell_{3}^{-}\right), & z \in R_{33} \\ \left(\ell_{1}^{+}, \ell_{2}^{-}, \ell_{3}^{+}\right), & z \in R_{32} \\ \left(\ell_{1}^{-}, \ell_{2}^{-}, \ell_{3}^{+}\right), & z \in R_{22} \\ \left(\ell_{1}^{-}, \ell_{2}^{+}, \ell_{3}^{+}\right), & z \in R_{21} .\end{cases}
$$

REMARKS 3.1. 1. As $z$ moves from a region $R_{i j}$ to the counterclockwise neighboring region, two of the rays $\hat{\ell}_{j}$ remain the same and the third one changes direction. As a consequence, the set of the three rays $\left(\hat{\ell}_{1}, \hat{\ell}_{2}, \hat{\ell}_{3}\right)$ advances by an angle $\pi / 3$ clockwise. Thus after $z$ moves through all six regions, the three rays advance by an angle $6 \pi / 3=2 \pi$, and they return to their starting position. 


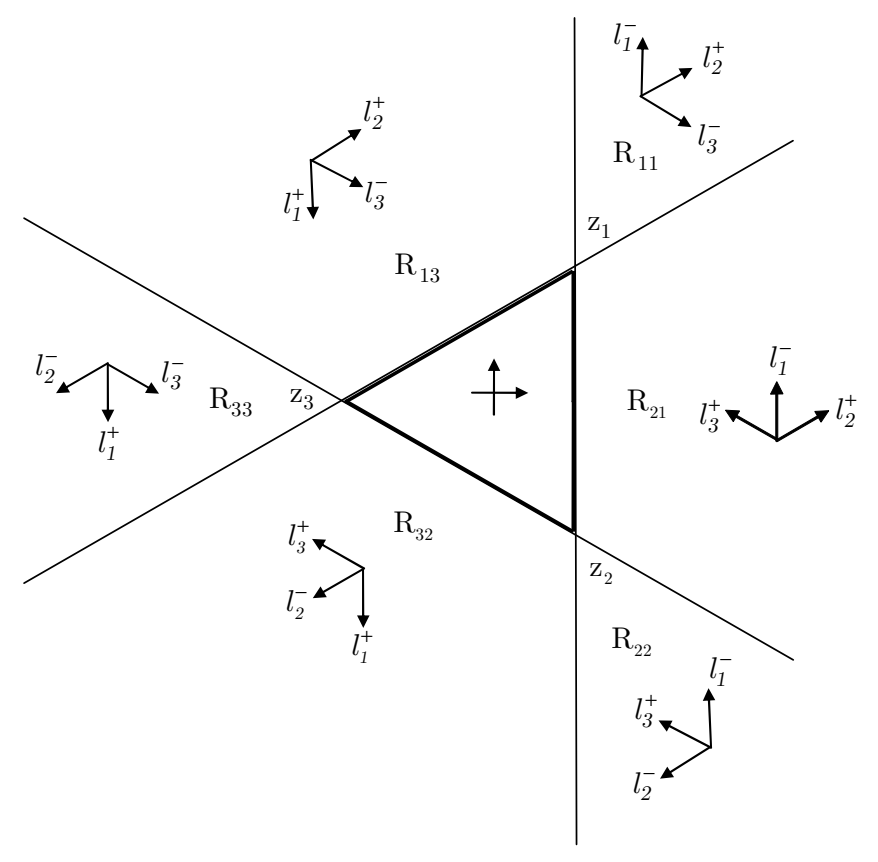

FIG. 3.2

2. Comparing with the interior problem, where the three rays $\ell_{j}$ are symmetrically located, making an angle $2 \pi / 3$ between each other, for the exterior problem the angles between the rays are always $\pi / 3, \pi / 3$ and $4 \pi / 3$.

4. The global relation. Before deriving the set of global relations needed for the exterior of the triangle, we first establish some symmetry relations.

Proposition 4.1. Assume that there exists a unique function $q(x, y)$ which satisfies the Laplace equation in the exterior of an equilateral triangle, which decays to zero as $|x|+|y| \rightarrow \infty$, and which satisfies

$$
q_{j}(s)=f(s), j=1,2,3,-\frac{\ell}{2} \leq s \leq \frac{\ell}{2} .
$$

Then the function $Q(z)=q_{z}(z)$ satisfies the symmetry relation

$$
Q(z)=\alpha Q(\alpha z), \quad z \in \Omega^{(e)} .
$$

Furthermore, if $f(s)$ is symmetric (even) or antisymmetric (odd) with respect to the midpoint of the side, then $Q(z)$ satisfies the following additional relations:

$$
f(-s)=f(s) \Rightarrow \overline{Q(\bar{z})}=Q(z), \quad \text { and } \quad f(-s)=-f(s) \Rightarrow \overline{Q(\bar{z})}=-Q(z) .
$$

Proof. Cauchy's theorem implies

$$
Q(z)=-\frac{1}{2 \pi i}\left[\int_{z_{2}}^{z_{1}}+\int_{z_{1}}^{z_{3}}+\int_{z_{3}}^{z_{2}}\right] \frac{Q(\zeta)}{\zeta-z} d \zeta
$$


where $z_{1}, z_{2}, z_{3}$ are shown in Figure 3.1. On the side $S_{3}, \zeta(s)$ is given by the first of equations (3.1), and thus $d \zeta=i d s$. On the side $S_{2}, \zeta(s)$ is given by the second of equations (3.1), and thus $d \zeta=i \alpha d s$. Similarly for the side $S_{1}$ and hence equation (4.3) becomes

$$
Q(z)=-\frac{1}{2 \pi} \int_{-\ell / 2}^{\ell / 2}\left(\frac{Q(\tau(s))}{\tau(s)-z}+\frac{\alpha Q(\alpha \tau(s))}{\alpha \tau(s)-z}+\frac{\bar{\alpha} Q(\bar{\alpha} \tau(s))}{\bar{\alpha} \tau(s)-z}\right) d s
$$

with

$$
\tau(s)=\frac{\ell}{2 \sqrt{3}}+i s, \quad-\frac{\ell}{2} \leq s \leq \frac{\ell}{2} .
$$

Replacing in equation (4.4) $z$ by $\alpha z$ and multiplying the resulting equation by $\alpha$, we find

$$
\alpha Q(\alpha z)=-\frac{1}{2 \pi} \int_{-\ell / 2}^{\ell / 2}\left(\frac{Q(\tau(s))}{\bar{\alpha} \tau(s)-z}+\frac{\alpha Q(\alpha \tau(s))}{\tau(s)-z}+\frac{\bar{\alpha} Q(\bar{\alpha} \tau(s))}{\alpha \tau(s)-z}\right) d s .
$$

Equations (4.4) and (4.5) are consistent with equation (4.1), as well as with equation $Q(z)=\bar{\alpha} Q(\bar{\alpha} z)$ which follows from equation (4.1) by replacing $z$ with $\bar{\alpha} z$. If $f(s)=f(-s)$ (the even symmetric case), then the reflection of the triangle with respect to the $x$-axis leads to the same geometry and boundary data and hence, due to uniqueness of the exterior Dirichlet problem solution, it follows that

$$
q(z, \bar{z})=q(\bar{z}, z)
$$

Differentiating with respect to $z$ we obtain

$$
q_{z}(z, \bar{z})=q_{z}(\bar{z}, z)=\overline{\bar{q}}_{\bar{z}}(\bar{z}, z)=\bar{q}_{\bar{z}}(\bar{z}, z)
$$

where for the last equation we have used the fact that $q$ is real. The first and the last equation imply

$$
Q(z)=\overline{Q(\bar{z})}
$$

If $f(s)=-f(-s)$ (the odd symmetric case), reflection with respect to the $x$-axis yields a Dirichlet problem with opposite data. Then uniqueness of the solution and linearity imply

$$
q(z, \bar{z})=-q(\bar{z}, z)
$$

and hence

$$
Q(z)=-\overline{Q(\bar{z})}
$$

Proposition 4.2. Let the real function $q(x, y)$ satisfy Laplace's equation in the domain $\Omega^{(e)}$ exterior to the equilateral triangle depicted in Figure 3.1, and let the smooth function $f(s)$ be the prescribed Dirichlet data on each side of the triangle. Assume that there exists a unique solution $q(x, y)$ which has sufficient smoothness all the way to the boundary 


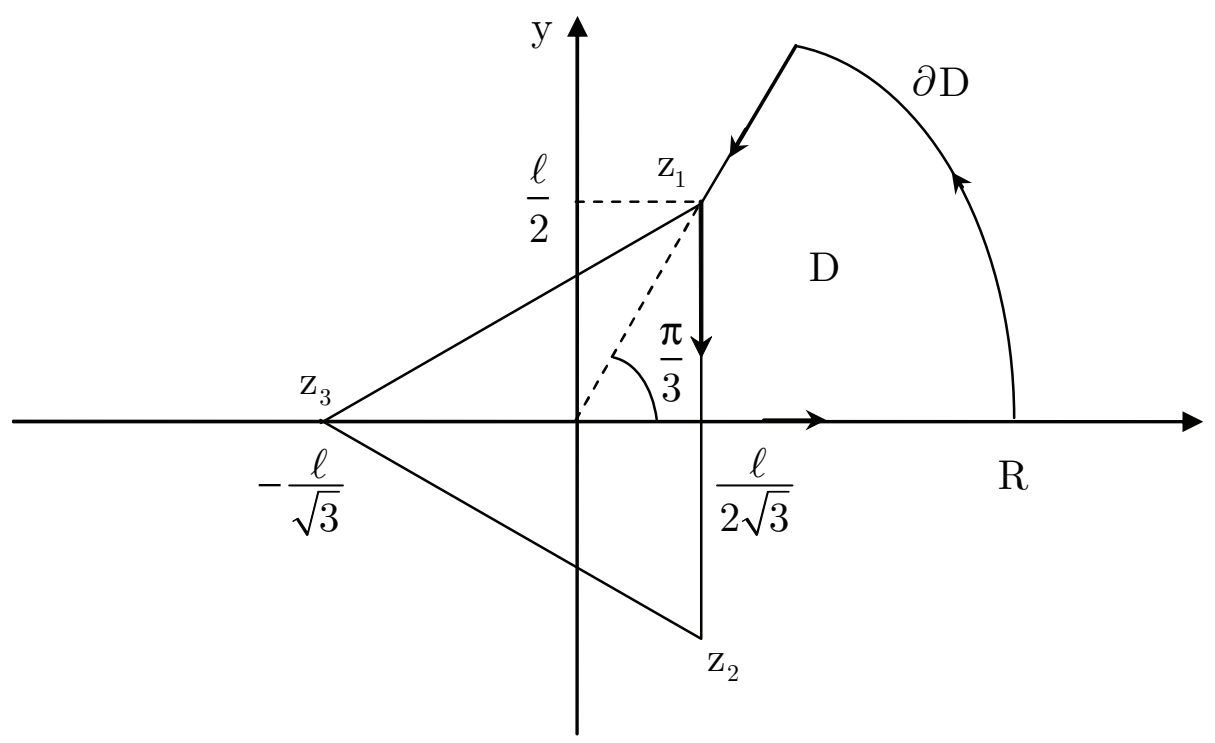

FIG. 4.1

and which vanishes at infinity. Then, the unknown functions

$$
\begin{aligned}
\Psi(k) & =\int_{0}^{\ell / 2} e^{k s} q_{N}(s) d s, \quad k \in \mathbb{C}, \\
G(k) & =\int_{0}^{+\infty} e^{k s} g\left(s+\frac{\ell}{\sqrt{3}}\right) d s, \quad \operatorname{Re} k \leq 0, \\
H(k) & =\int_{0}^{+\infty} e^{k s} h\left(s+\frac{\ell}{\sqrt{3}}\right) d s, \quad \operatorname{Re} k \leq 0,
\end{aligned}
$$

are coupled with the known function

$$
F(k)=\int_{0}^{\ell / 2} e^{k s} \dot{f}(s) d s, \quad k \in \mathbb{C}
$$

by the global relation

$$
i \Psi(k)+e^{\frac{k \ell}{2}} G(i \bar{\alpha} k)+H(-i k)=F(k), \quad-\pi \leq \arg k \leq-\frac{\pi}{3}
$$

where $q_{N}$ is the outward normal derivative of $q$ on the boundary of the triangle and $g, h$ are the following functions:

$$
h(s)=2 Q(s), \quad g(s)=2 Q\left(s e^{i \pi}\right), \quad 0<s<\infty .
$$


Proof. Let $D$ denote the domain depicted in Figure 4.1, where the boundary $\partial D$ is determined by the following contour

$$
\begin{aligned}
\partial D & =\left\{z=s \mid \frac{l}{2 \sqrt{3}} \leq s \leq R\right\} \cup\left\{z=\operatorname{Re}^{i \theta} \mid 0 \leq \theta \leq \frac{\pi}{3}\right\} \\
& \cup\left\{z=s e^{i \frac{\pi}{3}} \mid \frac{l}{\sqrt{3}} \leq s \leq R\right\} \cup\left\{z=\frac{l}{2 \sqrt{3}}+i s \mid 0 \leq s \leq \frac{l}{2}\right\} .
\end{aligned}
$$

Since $q$ satisfies Laplace's equation in $D$ it follows that

$$
\int_{\partial D} e^{-i k z} Q(z) d z=0, \quad Q=q_{z}
$$

For $R$ finite, equation (4.11) is valid for every $k \in \mathbb{C}$. However, as $R \rightarrow \infty$, using the fact that

$$
0 \leq \arg z \leq \frac{\pi}{3}
$$

it follows that equation (4.11) is valid for $k$ in the sector

$$
-\pi \leq \arg k \leq-\frac{\pi}{3}
$$

Equation (4.11) yields

$$
\begin{aligned}
& \int_{\ell / 2 \sqrt{3}}^{R} e^{-i k s} Q(s) d s+\int_{0}^{\pi / 3} e^{-i k R e^{i \theta}} Q\left(\operatorname{Re}^{i \theta}\right) i R e^{i \theta} d \theta \\
& +\int_{R}^{\ell / \sqrt{3}} e^{-i k s e^{i \frac{\pi}{3}}} Q\left(s e^{i \frac{\pi}{3}}\right) e^{i \frac{\pi}{3}} d s+\int_{\ell / 2}^{0} e^{-i k\left(\frac{\ell}{2 \sqrt{3}}+i s\right)} Q\left(\frac{\ell}{2 \sqrt{3}}+i s\right) i d s=0 .
\end{aligned}
$$

As $R \rightarrow \infty$, Jordan's Lemma implies that the second integral in (4.12) vanishes. Using

$$
Q\left(s e^{i \frac{\pi}{3}}\right)=\alpha Q\left(s \alpha e^{i \frac{\pi}{3}}\right)=\alpha Q\left(s e^{i \pi}\right)
$$

and

$$
Q\left(\frac{\ell}{2 \sqrt{3}}+i s\right)=\frac{1}{2} q_{x}(s)-\frac{i}{2} q_{y}(s)=-\frac{1}{2}\left(q_{N}(s)+i \dot{f}(s)\right)
$$

equation (4.12) becomes

$$
\begin{gathered}
\int_{\ell / 2 \sqrt{3}}^{\infty} e^{-i k s} Q(s) d s+\alpha e^{i \frac{\pi}{3}} \int_{\infty}^{\ell / \sqrt{3}} e^{-i k s e^{i \frac{\pi}{3}}} Q\left(s e^{i \pi}\right) d s \\
-\frac{1}{2} \int_{\ell / 2}^{0} e^{-i k\left(\frac{\ell}{2 \sqrt{3}}+i s\right)}\left(q_{N}(s)+i \dot{f}(s)\right) i d s=0 .
\end{gathered}
$$

Let

$$
\begin{aligned}
& 2 Q(s)=h(s), \quad s>\frac{\ell}{2 \sqrt{3}} \\
& 2 Q\left(s e^{i \pi}\right)=g(s), \quad s>\frac{\ell}{\sqrt{3}} .
\end{aligned}
$$

Then equation (4.13) becomes

$$
i e^{-i k \frac{\ell}{2 \sqrt{3}}} \int_{0}^{\ell / 2} e^{k s}\left(q_{N}(s)+i \dot{f}(s)\right) d s+\int_{\ell / \sqrt{3}}^{+\infty} e^{-i k s e^{i \frac{\pi}{3}}} g(s) d s+\int_{\ell / 2 \sqrt{3}}^{+\infty} e^{-i k s} h(s) d s=0 .
$$




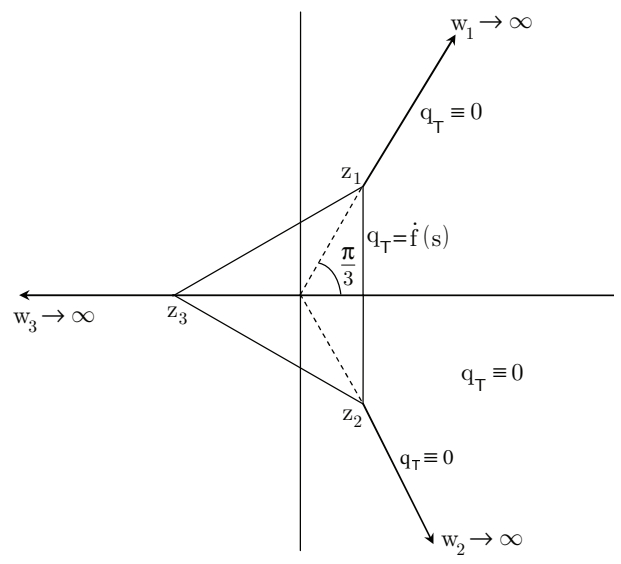

FIG. 4.2

Inserting (4.6)-(4.9) in (4.15) we arrive at the global relation (4.10).

REMARK 4.1. According to the second of equations (4.14) the functions $g, h$ assume real values in the even symmetric cases and imaginary values in the odd symmetric case. It turns out that the case of odd Dirichlet data leads to a problem formulated in the interior of a convex polygon formed by three sides.

TheOREm 4.2. Let the real function $q(x, y)$ satisfy Laplace's equation in the domain $\Omega^{(e)}$ exterior to the equilateral triangle depicted in Figure 3.1, and let the odd smooth function $f(s)$ be the prescribed Dirichlet data on each side of the triangle. Then the Neumann values $q_{N}(s)$ on each side of the triangle can be obtained by solving the Laplace equation in the domain $\Omega$ depicted in Figure 4.1 with the contour $w_{1} \rightarrow \infty, z_{1}, z_{2}, w_{2} \rightarrow \infty$ and with the following Dirichlet boundary conditions:

$$
q_{T}(s)=\dot{f}(s), \quad-\frac{\ell}{2} \leq s \leq \frac{\ell}{2}
$$

on $S_{3}, q_{T}=0$ on the sides $\left(z_{1}, w_{1}\right)$ and $\left(z_{2}, w_{2}\right)$.

Proof. The second of equations (4.2) applied on $z=x,-\infty<x<-\ell / \sqrt{3}$ (see Figure 4.2) yields

$$
Q(x)+\overline{Q(x)}=0, \quad-\infty<x<-\ell / \sqrt{3} .
$$

Hence,

$$
q_{x}(x, 0)=0, \quad-\infty<x<-\ell / \sqrt{3} .
$$

Therefore, the symmetry relations (4.1) and $Q(z)=\bar{\alpha} Q(\bar{\alpha} z)$ imply that $q_{T}=0$ on the rays $\left(z_{1}, w_{1} \rightarrow \infty\right)$ and $\left(z_{2}, w_{2} \rightarrow \infty\right)$. That completes the proof of the theorem.

REmark 4.3. A technique for the numerical evaluation of the Dirichlet to Neumann map for the Laplace equation in the interior of a convex polygon is presented in [8, 9]. Using this technique it is straightforward to evaluate the Neumann values $q_{N}(s)$ on the side $z_{1} z_{2}$ in terms of $\dot{f}(s)$. 


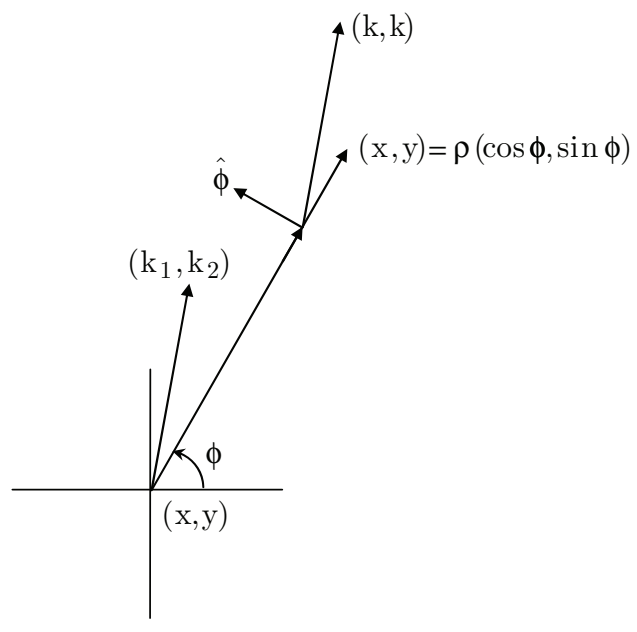

FIG. A.1

Appendix. We will show that

$$
\frac{1}{2 \pi} \iint_{\mathbb{R}^{2}} \frac{e^{i k_{1} x+i k_{2} y}}{i k_{1}-k_{2}} d k_{1} d k_{2}=\frac{1}{x+i y} .
$$

We introduce the polar system $\widehat{\boldsymbol{\rho}}, \widehat{\boldsymbol{\phi}}$ (see Figure A.1)

$$
x=\rho \cos \phi, \quad y=\rho \sin \phi .
$$

The vector $\widehat{\mathbf{k}}$ has the coordinates $\left(k_{1}, k_{2}\right)$ in the system $\widehat{\boldsymbol{x}}, \widehat{\boldsymbol{y}}$ and the coordinates $\left(k_{I}, k_{I I}\right)$ in the system $\widehat{\boldsymbol{\rho}}, \widehat{\boldsymbol{\phi}}$. The polar base vectors are

$$
\widehat{\boldsymbol{\rho}}=(\cos \phi, \sin \phi), \quad \widehat{\boldsymbol{\phi}}=(-\sin \phi, \cos \phi) .
$$

Since $\widehat{\boldsymbol{\rho}}, \widehat{\phi}$ are obtained via a rotation of the Cartesian system by an angle $+\phi$, we have that

$$
\left[\begin{array}{l}
k_{1} \\
k_{2}
\end{array}\right]=\left[\begin{array}{cc}
\cos \phi & -\sin \phi \\
\sin \phi & \cos \phi
\end{array}\right]\left[\begin{array}{c}
k_{I} \\
k_{I I}
\end{array}\right]
$$

Also,

$$
\begin{aligned}
& i k_{1} x+i k_{2} y=i \rho k_{I} \\
& i k_{1}-i k_{2}=\left(k_{I}+i k_{I I}\right) i e^{i \phi}
\end{aligned}
$$

which imply that

$$
\begin{aligned}
d k_{1} d k_{2}= & d k_{1} \wedge d k_{2}=\left(\cos \phi d k_{I}-\sin \phi d k_{I I}\right) \wedge\left(\sin \phi d k_{I}+\cos \phi d k_{I I}\right) \\
& =\cos ^{2} \phi d k_{I} \wedge d k_{I I}-\sin ^{2} \phi d k_{I I} \wedge d k_{I}=d k_{I} \wedge d k_{I I}=d k_{I} d k_{I I} .
\end{aligned}
$$

Formula (A.5) is a consequence of the fact that rotations leave the Lebesque measure invariant. Using (A.3), (A.4) and (A.5) we obtain

$$
\frac{1}{2 \pi} \iint_{\mathbb{R}^{2}} \frac{e^{i k_{1} x+i k_{2} y}}{i k_{1}-k_{2}} d k_{1} d k_{2}=\frac{e^{-i \phi}}{2 \pi i} \iint_{\mathbb{R}^{2}} \frac{e^{i \rho k_{I}}}{k_{I}+i k_{I I}} d k_{I} d k_{I I} .
$$


Since $\rho>0$, complex integration in the lower half plane implies

$$
\int_{-\infty}^{+\infty} \frac{e^{i \rho k_{I}}}{k_{I}+i k_{I I}} d k_{I}= \begin{cases}2 \pi i e^{\rho k_{I I}} & k_{I I}<0 \\ \pi i, & k_{I I}=0 \\ 0, & k_{I I}>0\end{cases}
$$

Hence, from (A.6) we obtain

$$
\frac{e^{-i \theta}}{2 \pi i} \iint_{\mathbb{R}^{2}} \frac{e^{i \rho k_{I}}}{k_{I}+i k_{I I}} d k_{I} d k_{I I}=\frac{e^{-i \phi}}{\rho} \int_{0}^{+\infty} e^{-t} d t=\frac{e^{-i \phi}}{\rho}=\frac{1}{x+i y} .
$$

\section{REFERENCES}

[1] G. Dassios and A.S. Fokas, "The Basic Elliptic Equations in an Equilateral Triangle", Proceedings of the Royal Society of London A, 461, pp. 2721-2748, 2005. MR2165508 (2006f:35048)

[2] A.S. Fokas and A.A. Kapaev, "A Riemann-Hilbert Approach to the Laplace Equation", Journal of Mathematical Analysis and Applications, 251, pp. 770-804, 2000. MR.1794770 (2001k:35241)

[3] A.S. Fokas, "A Unified Transform Method for Solving Linear and Nonlinear PDE's", Proceeding of the Royal Society of London A, 453, pp. 1411-1443, 1997. MR1469927(98e:35007)

[4] A.S. Fokas and A.A. Kapaev, "On a Transform Method for the Laplace Equation in polygon", IMA Journal in Applied Mathematics, 68, pp. 355-408, 2003. MR.1988152 (2004c:37176)

[5] A.S. Fokas, "Two-Dimensional Linear Partial Differential Equations in a Convex Polygon", Proceeding of the Royal Society of London A, 457, pp. 371-393, 2001. MR1848093 (2002j:35084)

[6] A.S. Fokas and M. Zyskin, "The Fundamental Differential Form and Boundary-Value Problems", Quarterly Journal of Mechanics and Applied Mathematics, 55, pp. 457-479, 2002. MR1919978 (2003f:35267)

[7] S.R. Fulton, A.S. Fokas and C.A. Xenophontos, "An Analytical Method for Linear Elliptic PDEs and its Numerical Implementation", Journal of Computational and Applied Mathematics, 167, pp. 465-483, 2004. MR2064703 (2005d:65216)

[8] A.G. Sifalakis, A.S. Fokas, S.R. Fulton and Y.G. Saridakis, "The Generalized Dirichlet-Neumann Map for Linear Elliptic PDEs and its Numerical Implementation", J. Comp. Appl. Math, 219, 9-34, 2008. MR2437692

[9] S.A. Smitheman, E.A. Spence and A.S. Fokas, "A spectral collocation method for the Laplace and Modified Helmholtz equations in a convex polygon", IMA J. Num. Anal. doi:10.1093/imanum/dm079 